\title{
Pulse Control of Decoherence with Population Decay
}

\author{
Takahiro MuraKami* and Yositake TAKAnE \\ Department of Quantum Matter, ADSM, Hiroshima University, 1-3-1 Kagamiyama, \\ Higashi-Hiroshima, Hiroshima 739-8530 \\ (Received )
}

\begin{abstract}
The pulse control of decoherence in a qubit interacting with a quantum environment is studied with focus on a general case where decoherence is induced by both pure dephasing and population decay. To observe how the decoherence is suppressed by periodic $\pi$ pulses, we present a simple method to calculate the time evolution of a qubit under arbitrary pulse sequences consisting of bit-flips and/or phase-flips. We examine the effectiveness of the two typical sequences: bb sequence consisting of only bit-flips, and bp sequence consisting of both bit- and phase-flips. It is shown that the effectiveness of the pulse sequences depends on a relative strength of the two decoherence processes especially when a pulse interval is slightly shorter than qubit-environment correlation times. In the short-interval limit, however, the bp sequence is always more effective than, or at least as effective as, the bb sequence.
\end{abstract}

KEYWORDS: decoherence, pure dephasing, population decay, qubit

\section{Introduction}

To employ a two-level quantum system as a qubit for quantum computations, a quantum superposed state prepared in it must be well preserved for a long time. However, since any quantum systems interact with their surrounding environment, a superposed state is degraded by getting entangled with an environment. The degradation of a quantum superposed state is called decoherence. To perform reliable quantum computations, we thus need to suppress decoherence caused by an environment. Several schemes to suppress decoherence have been proposed so far. ${ }^{1-8}$ Among them, we focus on the decoherence suppression scheme which uses a sequence of short $\pi$ pulses $^{6,7,9-15}$ since it is relatively easy to implement in experimental situations and can be readily applied to multi-qubit systems. We call this scheme the pulse control of decoherence. The pulse control has been proposed by Ban, ${ }^{6}$ and by Viola and Lloyd. ${ }^{7}$ They considered a qubit coupled with an environment assuming that qubit-environment interactions induce decoherence without population decay (i.e., pure dephasing). They calculated the time evolution of a qubit under periodic $\pi$ pulses, and showed that the pure dephasing can be suppressed if the pulse interval is much shorter than the correlation time for qubitenvironment interactions. It has been proposed that we can even suppress the decoherence

\footnotetext{
*E-mail address: m1279028@hiroshima-u.ac.jp
} 
with population decay by applying $\pi$ pulses..$^{9,10}$

Let us consider a qubit linearly interacting with a boson environment. The Hamiltonian is $\mathcal{H}_{0}=\mathcal{H}_{\mathrm{S}}+\mathcal{H}_{\mathrm{B}}+\mathcal{H}_{\mathrm{SB}}$ with

$$
\begin{aligned}
\mathcal{H}_{\mathrm{S}} & =\hbar \omega_{0} \frac{\sigma_{z}}{2} \otimes 1_{\mathrm{B}}, \\
\mathcal{H}_{\mathrm{B}} & =1_{\mathrm{S}} \otimes \sum_{k} \hbar \omega_{k} b_{k}^{\dagger} b_{k} .
\end{aligned}
$$

Here, $\mathcal{H}_{\mathrm{S}}$ and $\mathcal{H}_{\mathrm{B}}$ describe the qubit and the environment, respectively, and $\mathcal{H}_{\mathrm{SB}}$ describes their mutual interactions. We have used the pseudo-spin representation in expressing the qubit. Ban, and Viola and Lloyd, considered the case of $\mathcal{H}_{\mathrm{SB}}=\mathcal{H}_{z}$ with

$$
\mathcal{H}_{z}=\sum_{k} \hbar\left(g_{k z}^{*} \sigma_{z} \otimes b_{k}^{\dagger}+g_{k z} \sigma_{z} \otimes b_{k}\right)
$$

which induces the pure dephasing. ${ }^{6,7}$ They pointed out that the dephasing can be suppressed by the sequence of periodic $\pi$ pulses about the $x$-axis in the pseudo-spin space. They confirmed that the dephasing is notably suppressed if the interval $\Delta t$ between $\pi$ pulses is much shorter than the correlation time for qubit-environment interactions. Uchiyama and Aihara ${ }^{12}$ extended the argument to a nonlinear interaction case by using a diagrammatic perturbation method. However, we should note that the decoherence arises from not only pure dephasing but also population decay. A simple but standard model which can describe the decoherence with population decay is given by $\mathcal{H}_{\mathrm{SB}}=\mathcal{H}_{ \pm}$with

$$
\mathcal{H}_{ \pm}=\sum_{k} \hbar\left(g_{k \pm}^{*} \sigma_{-} \otimes b_{k}^{\dagger}+g_{k \pm} \sigma_{+} \otimes b_{k}\right) .
$$

It has been proposed that decoherence as well as population decay can be suppressed by applying periodic $\pi$ pulses about the $z$-axis if the interval $\Delta t$ is much shorter than the correlation time. ${ }^{9,10}$ The present authors ${ }^{14}$ confirmed this by numerically calculating the time evolution of the reduced density matrix for a qubit based on the time-convolutionless projection operator approach. Protopopescu ${ }^{13}$ reported a numerical study on the subject similar to this. However, they employed the approximation in which the commutator $\left[\mathcal{H}_{\mathrm{p}}(t), \mathcal{H}_{ \pm}\right]\left(\mathcal{H}_{\mathrm{p}}(t)\right.$ : external pulse field) is completely neglected. We have no reason to justify the approximation.

We focus on the case with

$$
\mathcal{H}_{\mathrm{SB}}=\mathcal{H}_{z}+\mathcal{H}_{ \pm}
$$

This case has not been studied extensively. This interaction Hamiltonian $\mathcal{H}_{\text {SB }}$ induces not only the pure dephasing but also the decoherence with population decay, and describes more general situations than those treated in refs. 6, 7 and 14. Each $\pi$ pulse about the $x$-axis $(z$ axis) induces a bit-flip (phase-flip). Periodic bit-flips cancel out the influence of $\mathcal{H}_{z}$, while periodic phase-flips cancel out the influence of $\mathcal{H}_{ \pm}$. Let $\tau_{\mathrm{c} z}$ and $\tau_{\mathrm{c} \pm}$ be the correlation times for $\mathcal{H}_{z}$ and $\mathcal{H}_{ \pm}$, respectively. It has been shown that if $\Delta t$ is much shorter than $\tau_{\mathrm{c} z}$ and $\tau_{\mathrm{c} \pm}$, 
the decoherence caused by $\mathcal{H}_{\mathrm{SB}}=\mathcal{H}_{z}+\mathcal{H}_{ \pm}$can be suppressed by a sequence of both bit-flips and phase-flips. ${ }^{9,10}$ The simplest sequence is composed by the alternate application of a bitflip and a phase-flip. We call it bp sequence. Although its effectiveness has been proved in the short interval limit of $\Delta t \ll \tau_{\mathrm{c} z}$ and $\tau_{\mathrm{c} \pm}$, a realization of this condition is not necessarily possible in actual experiments. It is of interest to study the effectiveness in experimentally relevant cases of $\Delta t \lesssim \tau_{\mathrm{c} z}$ and $\tau_{\mathrm{c} \pm}$. Furthermore, we are also interested in whether the bp sequence is always more effective than the sequence consisting of only bit-flips. We call the latter one bb sequence hereafter. If $\tau_{\mathrm{c} z} \sim \tau_{\mathrm{c} \pm}$, we expect that the bp sequence is much more effective than the bb sequence. However, in the regime of $\tau_{\mathrm{c} z} \ll \tau_{\mathrm{c} \pm}$, the bb sequence may predominate the bp sequence.

In this paper, we study the time evolution of a qubit coupled with a quantum environment under periodic $\pi$ pulses, to examine the effectiveness of the bp and bb sequences when the interaction Hamiltonian is given by eq. (5). It is assumed that qubit-environment coupling is week. We employ the time-convolutionless projection operator approach following ref. 14, and derive the equation of motion which describes the time evolution of the reduced density matrix for a qubit. Our approach is simpler than that presented in ref. 12, and more accurate than that presented in ref. 13. Besides the approaches in refs. 12 and 13, several approximate methods based on the influence-functional formalism may be applicable. ${ }^{16}$ However, such methods are too complicated for our restricted purpose. The resulting equation has an advantage that it is applicable to arbitrary pulse sequences consisting of bit- and/or phase flips. We examine the three cases of $\tau_{\mathrm{c} \pm} / \tau_{\mathrm{c} z}=2,5$ and 50 for a fixed value of $\tau_{\mathrm{c}}$, where $\tau_{\mathrm{c}}$ is defined by $\tau_{\mathrm{c}}{ }^{-2} \equiv \tau_{\mathrm{c} z}{ }^{-2}+\tau_{\mathrm{c} \pm}{ }^{-2}$. The pulse interval is chosen as $\Delta t / \tau_{\mathrm{c}}=2^{-2}, 2^{-3}$ and $2^{-4}$. We show that the bp sequence is much more effective than the bb sequence when $\tau_{\mathrm{c} \pm} / \tau_{\mathrm{c} z}=2$. However, we also show that their relative effectiveness can be reversed in the cases of $\tau_{\mathrm{c} \pm} / \tau_{\mathrm{c} z}=5$ and 50 depending on $\Delta t / \tau_{\mathrm{c}}$. Only in the short-interval limit, the bp sequence is always more effective than, or at least as effective as, the bb sequence. We conclude that the effectiveness of the

pulse sequences depends on a relative strength of the two decoherence processes when a pulse interval is slightly shorter than $\tau_{\mathrm{c}}$.

\section{Formulation}

We derive the equation of motion for the reduced density matrix for a qubit. Let us consider the case where the qubit-environment interaction is given by eq. (5) and periodic $\pi$ pulses with an interval $\Delta t$ are applied. We also assume that the duration of each pulse is infinitely short. The bb sequence consisting of only bit-flips is described by

$$
\mathcal{H}_{\mathrm{bb}}(t)=\hbar \frac{\pi}{2} \sum_{j=1}^{\infty} \delta(t-j \Delta t)\left(\sigma_{x} \cos \omega_{0} t+\sigma_{y} \sin \omega_{0} t\right) \otimes 1_{\mathrm{B}}
$$


The bp sequence consisting of both bit- and phase-flips is described by

$$
\mathcal{H}_{\mathrm{bp}}(t)=\hbar \frac{\pi}{2} \sum_{j=1}^{\infty}\left[\delta(t-(2 j-1) \Delta t)\left(\sigma_{x} \cos \omega_{0} t+\sigma_{y} \sin \omega_{0} t\right)+\delta(t-2 j \Delta t) \sigma_{z}\right] \otimes 1_{\mathrm{B}},
$$

where a bit-flip is applied at $t=(2 j-1) \Delta t$ while a phase-flip is applied at $t=2 j \Delta t$. The total Hamiltonian is $\mathcal{H}(t) \equiv \mathcal{H}_{0}+\mathcal{H}_{\mathrm{p}}(t)$, where $\mathcal{H}_{\mathrm{p}}(t)$ represents either $\mathcal{H}_{\mathrm{bb}}(t)$ or $\mathcal{H}_{\mathrm{bp}}(t)$. Although we treat only the bb and bp sequences, the following argument can be applied to any pulse sequences consisting of bit- and/or phase-flips. Let $\rho(t)$ be the density matrix of the whole system. It obeys the Liouville-von Neumann equation

$$
\frac{\partial}{\partial t} \rho(t)=L(t) \rho(t)
$$

with

$$
L(t) \cdot=-\frac{\mathrm{i}}{\hbar}[\mathcal{H}(t), \cdot] .
$$

We decompose $\mathcal{H}(t)$ as $\mathcal{H}(t)=\mathcal{H}_{1}(t)+\mathcal{H}_{\mathrm{SB}}$, where $\mathcal{H}_{1}(t)=\mathcal{H}_{\mathrm{S}}+\mathcal{H}_{\mathrm{B}}+\mathcal{H}_{\mathrm{p}}(t)$. It is convenient to introduce $\tilde{\rho}(t)$ which is defined as

$$
\tilde{\rho}(t)=T_{\mathrm{a}} \mathrm{e}^{-\int_{0}^{t} d \tau L_{1}(\tau)} \rho(t),
$$

where $T_{\mathrm{a}}$ is the anti-time-ordering operator and $L_{1}(t) \cdot=-\frac{\mathrm{i}}{\hbar}\left[\mathcal{H}_{1}(t), \cdot\right]$. From eq. (8), we obtain

$$
\frac{\partial}{\partial t} \tilde{\rho}(t)=\tilde{L}_{\mathrm{SB}}(t) \tilde{\rho}(t)
$$

where $\tilde{L}_{\mathrm{SB}}(t) \cdot=-\frac{\mathrm{i}}{\hbar}\left[\tilde{\mathcal{H}}_{\mathrm{SB}}(t), \cdot\right]$. Here, $\tilde{\mathcal{H}}_{\mathrm{SB}}(t)$ is given by

$$
\begin{aligned}
\tilde{\mathcal{H}}_{\mathrm{SB}}(t) & =T_{\mathrm{a}} \mathrm{e}^{\frac{\mathrm{i}}{\hbar} \int_{0}^{t} d \tau \mathcal{H}_{1}(\tau)} \mathcal{H}_{\mathrm{SB}} T \mathrm{e}^{-\frac{\mathrm{i}}{\hbar} \int_{0}^{t} d \tau \mathcal{H}_{1}(\tau)} \\
& =T_{\mathrm{a}} \mathrm{e}^{\frac{\mathrm{i}}{\hbar} \int_{0}^{t} d \tau \hat{\mathcal{H}}_{\mathrm{p}}(\tau)} \hat{\mathcal{H}}_{\mathrm{SB}}(t) T \mathrm{e}^{-\frac{\mathrm{i}}{\hbar} \int_{0}^{t} d \tau \hat{\mathcal{H}}_{\mathrm{p}}(\tau)}
\end{aligned}
$$

where $T$ is the time-ordering operator, $\hat{\mathcal{H}}_{\mathrm{p}}(t)=\mathrm{e}^{\frac{\mathrm{i}}{\hbar}\left(\mathcal{H}_{\mathrm{S}}+\mathcal{H}_{\mathrm{B}}\right) t} \mathcal{H}_{\mathrm{p}}(t) \mathrm{e}^{-\frac{\mathrm{i}}{\hbar}\left(\mathcal{H}_{\mathrm{S}}+\mathcal{H}_{\mathrm{B}}\right) t}$ and

$$
\begin{aligned}
\hat{\mathcal{H}}_{\mathrm{SB}}(t)= & \mathrm{e}^{\frac{\mathrm{i}}{\hbar}\left(\mathcal{H}_{\mathrm{S}}+\mathcal{H}_{\mathrm{B}}\right) t} \mathcal{H}_{\mathrm{SB}} \mathrm{e}^{-\frac{\mathrm{i}}{\hbar}\left(\mathcal{H}_{\mathrm{S}}+\mathcal{H}_{\mathrm{B}}\right) t} \\
= & \hbar \sum_{k}\left(g_{k z}^{*} \sigma_{z} \otimes b_{k}^{\dagger} \mathrm{e}^{\mathrm{i} \omega_{k} t}+g_{k z} \sigma_{z} \otimes b_{k} \mathrm{e}^{-\mathrm{i} \omega_{k} t}\right) \\
& +\hbar \sum_{k}\left(g_{k \pm}^{*} \sigma_{-} \otimes b_{k}^{\dagger} \mathrm{e}^{\mathrm{i}\left(\omega_{k}-\omega_{0}\right) t}+g_{k \pm} \sigma_{+} \otimes b_{k} \mathrm{e}^{-\mathrm{i}\left(\omega_{k}-\omega_{0}\right) t}\right) .
\end{aligned}
$$

We can show that the time-ordered exponential factor in eq. (12) is obtained as

$$
T \mathrm{e}^{-\frac{\mathrm{i}}{\hbar} \int_{0}^{t} d \tau \hat{\mathcal{H}}_{\mathrm{p}}(\tau)}= \begin{cases}1_{\mathrm{S}} \otimes 1_{\mathrm{B}} & \text { for } 0<t<\Delta t, \\ \mathrm{i} \sigma_{x} \otimes 1_{\mathrm{B}} & \text { for } \Delta t<t<2 \Delta t, \\ \left(\mathrm{i} \sigma_{x} \times \mathrm{i} \sigma_{x}\right) \otimes 1_{\mathrm{B}} & \text { for } 2 \Delta t<t<3 \Delta t, \\ \left(\mathrm{i} \sigma_{x} \times \mathrm{i} \sigma_{x} \times \mathrm{i} \sigma_{x}\right) \otimes 1_{\mathrm{B}} & \text { for } 3 \Delta t<t<4 \Delta t, \\ \cdots & \cdots\end{cases}
$$


for $\mathcal{H}_{\mathrm{p}}(t)=\mathcal{H}_{\mathrm{bb}}(t)$ and

$$
T \mathrm{e}^{-\frac{\mathrm{i}}{\hbar} \int_{0}^{t} d \tau \hat{\mathcal{H}}_{\mathrm{p}}(\tau)}= \begin{cases}1_{\mathrm{S}} \otimes 1_{\mathrm{B}} & \text { for } 0<t<\Delta t, \\ \mathrm{i} \sigma_{x} \otimes 1_{\mathrm{B}} & \text { for } \Delta t<t<2 \Delta t, \\ \left(\mathrm{i} \sigma_{z} \times \mathrm{i} \sigma_{x}\right) \otimes 1_{\mathrm{B}} & \text { for } 2 \Delta t<t<3 \Delta t, \\ \left(\mathrm{i} \sigma_{x} \times \mathrm{i} \sigma_{z} \times \mathrm{i} \sigma_{x}\right) \otimes 1_{\mathrm{B}} & \text { for } 3 \Delta t<t<4 \Delta t, \\ \cdots & \cdots\end{cases}
$$

for $\mathcal{H}_{\mathrm{p}}(t)=\mathcal{H}_{\mathrm{bp}}(t)$. Let $N_{x}(t)\left(N_{z}(t)\right)$ be the number of bit-flips (phase-flips) within [0,t]. If the bb sequence is applied (i.e., $\mathcal{H}_{\mathrm{p}}(t)=\mathcal{H}_{\mathrm{bb}}(t)$ ), we find that

$$
\begin{aligned}
& N_{x}(t)=\left[\frac{t}{\Delta t}\right], \\
& N_{z}(t)=0,
\end{aligned}
$$

where $[a]$ denotes the integral part of the real number $a$. In the case where the bp sequence is applied, we find that

$$
\begin{aligned}
& N_{x}(t)=\left[\frac{t+\Delta t}{2 \Delta t}\right], \\
& N_{z}(t)=\left[\frac{t}{2 \Delta t}\right] .
\end{aligned}
$$

If we define

$$
\sigma_{\mathrm{b}}(t)= \begin{cases}\sigma_{+} & \text {if } N_{x}(t) \text { is even } \\ \sigma_{-} & \text {if } N_{x}(t) \text { is odd }\end{cases}
$$

we obtain

$$
\tilde{\mathcal{H}}_{\mathrm{SB}}(t)=(-1)^{N_{x}(t)} \hat{\mathcal{H}}_{z}(t)+(-1)^{N_{z}(t)} \hat{\mathcal{H}}_{ \pm}(t),
$$

where

$$
\hat{\mathcal{H}}_{z}(t)=\hbar \sum_{k}\left(g_{k z}^{*} \sigma_{z} \otimes b_{k}^{\dagger} \mathrm{e}^{\mathrm{i} \omega_{k} t}+g_{k z} \sigma_{z} \otimes b_{k} \mathrm{e}^{-\mathrm{i} \omega_{k} t}\right)
$$

and

$$
\hat{\mathcal{H}}_{ \pm}(t)=\hbar \sum_{k}\left(g_{k \pm}^{*} \sigma_{\mathrm{b}}^{\dagger}(t) \otimes b_{k}^{\dagger} \mathrm{e}^{\mathrm{i}\left(\omega_{k}-\omega_{0}\right) t}+g_{k \pm} \sigma_{\mathrm{b}}(t) \otimes b_{k} \mathrm{e}^{-\mathrm{i}\left(\omega_{k}-\omega_{0}\right) t}\right)
$$

Equation (21) holds for any pulse sequences consisting of bit- and/or phase-flips, although we have assumed that $\mathcal{H}_{\mathrm{p}}(t)$ is equal to $\mathcal{H}_{\mathrm{bb}}(t)$ or $\mathcal{H}_{\mathrm{bp}}(t)$. Note that each bit-flip $\pi$ pulse changes the sign of $\hat{\mathcal{H}}_{z}(t)$ and each phase-flip $\pi$ pulse changes the sign of $\hat{\mathcal{H}}_{ \pm}(t)$. The periodic sign change due to bit-flips (phase-flips) effectively cancels out the qubit-environment interaction $\hat{\mathcal{H}}_{z}(t)\left(\hat{\mathcal{H}}_{ \pm}(t)\right)$. This is the reason why the decoherence is suppressed by the $\pi$ pulses.

Our interest is focused on the time evolution of the qubit, which is described by the reduced density matrix defined by

$$
\tilde{\rho}_{\mathrm{S}}(t)=\operatorname{Tr}_{\mathrm{B}}\{\tilde{\rho}(t)\}=\left(\begin{array}{cc}
\tilde{\rho}_{11}(t) & \tilde{\rho}_{10}(t) \\
\tilde{\rho}_{01}(t) & \tilde{\rho}_{00}(t)
\end{array}\right),
$$


J. Phys. Soc. Jpn.

Full PAPER

where $\tilde{\rho}_{11}\left(\tilde{\rho}_{00}\right)$ represents the population in the upper (lower) state and the off-diagonal terms characterize the coherence of the qubit. We derive the equation of motion for $\tilde{\rho}_{\mathrm{S}}(t)$ following ref. 14. In doing so, we assume that the qubit and the environment are uncorrelated at $t=0$, i.e.,

$$
\tilde{\rho}(0)=\tilde{\rho}_{\mathrm{S}}(0) \otimes \tilde{\rho}_{\mathrm{B}}(0),
$$

and the environment is initially in thermal equilibrium at temperature $T$, i.e.,

$$
\tilde{\rho}_{\mathrm{B}}(0)=\rho_{\mathrm{B}}=\prod_{k}\left(1-\mathrm{e}^{-\beta \hbar \omega_{k}}\right) \mathrm{e}^{-\beta \hbar \omega_{k} b_{k}^{\dagger} b_{k}}
$$

with $\beta=1 / k_{\mathrm{B}} T$ ( $k_{\mathrm{B}}$ : the Boltzmann constant). We introduce the projection operator $P$ which is defined as $P \cdot=\operatorname{Tr}_{\mathrm{B}}\{\cdot\} \otimes \rho_{\mathrm{B}}$. We observe that $P \tilde{\rho}(t)=\tilde{\rho}_{\mathrm{S}}(t) \otimes \rho_{\mathrm{B}}$. Adapting the timeconvolutionless projection operator formalism ${ }^{17}$ with $P$ to eq. (11), we derive the equation of motion for $\tilde{\rho}_{\mathrm{S}}(t)$,

$$
\frac{\partial}{\partial t} \tilde{\rho}_{\mathrm{S}}(t)=\operatorname{Tr}_{\mathrm{B}}\left\{\int_{0}^{t} d t_{1} \tilde{L}_{\mathrm{SB}}(t) \tilde{L}_{\mathrm{SB}}\left(t_{1}\right)\left[\tilde{\rho}_{\mathrm{S}}(t) \otimes \rho_{\mathrm{B}}\right]\right\}
$$

where we have neglected higher-order terms by assuming that the qubit-environment interaction is weak enough. In calculating the right-hand side of eq. (27), we note that the decoherence without population decay is mainly caused by the modes with $\omega_{k} \approx 0$ in $\hat{\mathcal{H}}_{z}(t)$ and the decoherence with population decay is mainly caused by the modes with $\omega_{k} \approx \omega_{0}$ in $\hat{\mathcal{H}}_{ \pm}(t)$. Thus, we approximately neglect the cross terms between $\hat{\mathcal{H}}_{z}(t)$ and $\hat{\mathcal{H}}_{ \pm}(t)$ in eq. (27).

Using eqs. (13) and (21), we obtain

$$
\begin{aligned}
\frac{\partial}{\partial t} \tilde{\rho}_{11}(t) & =-\gamma_{11}(t) \tilde{\rho}_{11}(t)+\eta_{11}(t) \\
\frac{\partial}{\partial t} \tilde{\rho}_{10}(t) & =-\gamma_{10}^{\Re}(t) \operatorname{Re}\left[\tilde{\rho}_{10}(t)\right]-\mathrm{i} \gamma_{10}^{\Im}(t) \operatorname{Im}\left[\tilde{\rho}_{10}(t)\right],
\end{aligned}
$$

where

$$
\begin{aligned}
\gamma_{11}(t)= & 2 \sum_{k}\left|g_{k \pm}\right|^{2} \operatorname{coth}\left(\frac{\hbar \omega_{k}}{2 k_{\mathrm{B}} T}\right) \int_{0}^{t} d t_{1}(-1)^{N_{z}(t)+N_{z}\left(t_{1}\right)} \\
& \times \frac{1+(-1)^{N_{x}(t)+N_{x}\left(t_{1}\right)}}{2} \cos \left(\omega_{k}-\omega_{0}\right)\left(t_{1}-t\right), \\
\eta_{11}(t)= & \sum_{k}\left|g_{k \pm}\right|^{2}\left[\operatorname{coth}\left(\frac{\hbar \omega_{k}}{2 k_{\mathrm{B}} T}\right)-(-1)^{N_{x}(t)}\right] \int_{0}^{t} d t_{1}(-1)^{N_{z}(t)+N_{z}\left(t_{1}\right)} \\
& \times \frac{1+(-1)^{N_{x}(t)+N_{x}\left(t_{1}\right)}}{2} \cos \left(\omega_{k}-\omega_{0}\right)\left(t_{1}-t\right), \\
\gamma_{10}^{\Re}(t)= & 4 \sum_{k}\left|g_{k z}\right|^{2} \operatorname{coth}\left(\frac{\hbar \omega_{k}}{2 k_{\mathrm{B}} T}\right) \int_{0}^{t} d t_{1}(-1)^{N_{x}(t)+N_{x}\left(t_{1}\right)} \cos \omega_{k}\left(t_{1}-t\right) \\
& +\sum_{k}\left|g_{k \pm}\right|^{2} \operatorname{coth}\left(\frac{\hbar \omega_{k}}{2 k_{\mathrm{B}} T}\right) \int_{0}^{t} d t_{1}(-1)^{N_{z}(t)+N_{z}\left(t_{1}\right)} \\
& \times(-1)^{N_{x}(t)+N_{x}\left(t_{1}\right)} \mathrm{e}^{\mathrm{i}(-1)^{N_{x}(t)}\left(\omega_{k}-\omega_{0}\right)\left(t_{1}-t\right)}
\end{aligned}
$$





Fig. 1. Time evolution of $\tilde{\rho}_{11}$ as a function of $t / \tau_{\mathrm{c}}$ at $k_{\mathrm{B}} T / \hbar \omega_{\mathrm{c}}=10^{-3}$. The ratio of the qubitenvironment correlation times is $\tau_{\mathrm{c} \pm} / \tau_{\mathrm{c} z}=2$. The pulse interval $\Delta t$ is (a) $\Delta t / \tau_{\mathrm{c}}=2^{-3}$ and (b) $2^{-4}$. The solid lines and dotted lines correspond to the cases of the bb sequence and the bp sequence, respectively. The dashed lines represent the case where $\pi$ pulses are absent.

$$
\begin{aligned}
\gamma_{10}^{\Im}(t)= & 4 \sum_{k}\left|g_{k z}\right|^{2} \operatorname{coth}\left(\frac{\hbar \omega_{k}}{2 k_{\mathrm{B}} T}\right) \int_{0}^{t} d t_{1}(-1)^{N_{x}(t)+N_{x}\left(t_{1}\right)} \cos \omega_{k}\left(t_{1}-t\right) \\
& +\sum_{k}\left|g_{k \pm}\right|^{2} \operatorname{coth}\left(\frac{\hbar \omega_{k}}{2 k_{\mathrm{B}} T}\right) \int_{0}^{t} d t_{1}(-1)^{N_{z}(t)+N_{z}\left(t_{1}\right)} \\
& \times \mathrm{e}^{\mathrm{i}(-1)^{N_{x}(t)}\left(\omega_{k}-\omega_{0}\right)\left(t_{1}-t\right)}
\end{aligned}
$$

In deriving eqs. (28) and (29), we have used the two relations $\tilde{\rho}_{11}(t)+\tilde{\rho}_{00}(t)=1$ and $\tilde{\rho}_{10}(t)=$ $\tilde{\rho}_{01}^{*}(t)$. Equations $(28)$ and $(29)$ describe the time evolution of the qubit under $\pi$ pulses. It should be emphasized that the above equation can be applied to any pulse sequences consisting of bit- and/or phase-flips. Introducing the spectral functions $I_{z}(\omega)$ and $I_{ \pm}(\omega)$ defined by

$$
\begin{aligned}
I_{z}(\omega) & =\sum_{k}\left|g_{k z}\right|^{2} \delta\left(\omega-\omega_{k}\right), \\
I_{ \pm}(\omega) & =\sum_{k}\left|g_{k \pm}\right|^{2} \delta\left(\omega-\omega_{k}\right),
\end{aligned}
$$

we rewrite eq. (33) as

$$
\begin{aligned}
\gamma_{10}^{\Im}(t)= & 4 \int_{0}^{\infty} d \omega I_{z}(\omega) \operatorname{coth}\left(\frac{\hbar \omega}{2 k_{\mathrm{B}} T}\right) \int_{0}^{t} d t_{1}(-1)^{N_{x}(t)+N_{x}\left(t_{1}\right)} \cos \omega\left(t_{1}-t\right) \\
& +\int_{0}^{\infty} d \omega I_{ \pm}(\omega) \operatorname{coth}\left(\frac{\hbar \omega}{2 k_{\mathrm{B}} T}\right) \int_{0}^{t} d t_{1}(-1)^{N_{z}(t)+N_{z}\left(t_{1}\right)} \mathrm{e}^{\mathrm{i}(-1)^{N_{x}(t)}\left(\omega-\omega_{0}\right)\left(t_{1}-t\right)}
\end{aligned}
$$

Equations (30), (31) and (32) are rewritten in the manner similar to eq. (36).

\section{Numerical Results}

On the basis of the resulting equation of motion, we study the time evolution of the reduced density matrix $\tilde{\rho}_{\mathrm{S}}(t)$ in the presence of periodic $\pi$ pulses with an interval $\Delta t$. We numerically calculate $\tilde{\rho}_{11}(t),\left|\tilde{\rho}_{10}(t)\right|$ and $\Delta \theta(t)$, where $\Delta \theta(t)$ is defined by $\Delta \theta(t)=$ 
$\arg \left\{\tilde{\rho}_{10}(t)\right\}-\arg \left\{\tilde{\rho}_{10}(0)\right\}$. We assume that the spectral function is given by

$$
\begin{aligned}
& I_{z}(\omega)=G_{z} \omega \mathrm{e}^{-\omega / \omega_{\mathrm{c}}} \\
& I_{ \pm}(\omega)=G_{ \pm} \omega \mathrm{e}^{-\omega / \omega_{\mathrm{c}}}
\end{aligned}
$$

where $\omega_{\mathrm{c}}$ is a cut-off frequency, and $G_{z}$ and $G_{ \pm}$are coupling constants. The following parameters are employed: $\omega_{0} / \omega_{\mathrm{c}}=0.1$ and $k_{\mathrm{B}} T /\left(\hbar \omega_{\mathrm{c}}\right)=0.001$. In terms of the coupling constants, the correlation times for $\mathcal{H}_{z}$ and $\mathcal{H}_{ \pm}$are given by $\tau_{\mathrm{c} z} \equiv\left(1 / 2 G_{z}\right)^{1 / 2} \omega_{\mathrm{c}}{ }^{-1}$ and $\tau_{\mathrm{c} \pm} \equiv\left(2 / G_{ \pm}\right)^{1 / 2} \omega_{\mathrm{c}}{ }^{-1}$, respectively. The initial behavior of $\tilde{\rho}_{10}(t)$ is expressed as $\tilde{\rho}_{10}(t)=\tilde{\rho}_{10}(0) \exp \left[-\left(t / \tau_{\mathrm{c}}\right)^{2}\right]$, where

$$
\frac{1}{\tau_{\mathrm{c}}^{2}} \equiv \frac{1}{\tau_{\mathrm{c} z}^{2}}+\frac{1}{\tau_{\mathrm{c} \pm}^{2}} \text {. }
$$

We fix $\tau_{\mathrm{c}}$ at $\tau_{\mathrm{c}}=0.4 \times\left(2 \pi / \omega_{\mathrm{c}}\right)$ and examine the cases of $\tau_{\mathrm{c} \pm} / \tau_{\mathrm{c} z}=2,5$ and 50 . While the pure dephasing is slightly stronger than the decoherence with population decay in the case of $\tau_{\mathrm{c} \pm} / \tau_{\mathrm{c} z}=2$, the former predominates the latter in the case of $\tau_{\mathrm{c} \pm} / \tau_{\mathrm{c} z}=50$. For each case, we calculate the time evolution with $\Delta t / \tau_{\mathrm{c}}=2^{-2}, 2^{-3}$ and $2^{-4}$. We choose $(|0\rangle+|1\rangle) / \sqrt{2}$ and $(|0\rangle+\mathrm{i}|1\rangle) / \sqrt{2}$ as initial states. From numerical results, we find that the decoherence suppression is relatively weak in the latter case. Thus, we present only the results for $(|0\rangle+$ i| 1$\rangle) / \sqrt{2}$ with

$$
\tilde{\rho}_{\mathrm{S}}(0)=\left(\begin{array}{cc}
1 / 2 & \mathrm{i} / 2 \\
-\mathrm{i} / 2 & 1 / 2
\end{array}\right) .
$$

In Fig. 1, we display $\tilde{\rho}_{11}(t)$ with (a) $\Delta t / \tau_{\mathrm{c}}=2^{-3}$ and (b) $2^{-4}$ in the case of $\tau_{\mathrm{c} \pm} / \tau_{\mathrm{c} z}=2$. We do not display the results for $\tau_{\mathrm{c} \pm} / \tau_{\mathrm{c} z}=5$ and 50 because variations of $\tilde{\rho}_{11}(t)$ are very small. The decrease of $\tilde{\rho}_{11}$ represents the population decay. We observe that the population decay is suppressed by either of the bb or bp sequences. It should be emphasized again that the bb sequence cannot suppress the influence of $\mathcal{H}_{ \pm}$, which induces population decay, while the influence is suppressed by the bp sequence containing phase-flip $\pi$ pulses. The suppression of the population decay under the bb sequence is simply attributed to periodic exchanges of the upper-state population with the lower-state population due to bit-flip $\pi$ pulses.

We compare $\tilde{\rho}_{10}(t)$ under the bp sequence with that under the bb sequence. In Fig. 2, we display $\left|\tilde{\rho}_{10}(t)\right|$ with $\Delta t / \tau_{\mathrm{c}}=2^{-2}, 2^{-3}$ and $2^{-4}$ when (a) $\tau_{\mathrm{c} \pm} / \tau_{\mathrm{c} z}=2$, (b) 5 and (c) 50 . The decrease of $\left|\tilde{\rho}_{10}(t)\right|$ represents the decoherence. Figure 2 shows that in the case of $\tau_{\mathrm{c} \pm} / \tau_{\mathrm{c} z}=2$, the decoherence suppression under the bp sequence is much more effective than that under the bb sequence. For the bb sequence, the decrease of $\Delta t$ from $\Delta t / \tau_{\mathrm{c}}=2^{-3}$ to $2^{-4}$ does not result in a notable improvement. This fact is attributed to a shortcoming of the bb sequence which cannot cancel out the influence of $\mathcal{H}_{ \pm}$. However, the effectiveness of the bb sequence is improved with increasing $\tau_{\mathrm{c} \pm} / \tau_{\mathrm{c} z}$ since the influence of $\mathcal{H}_{ \pm}$is relatively reduced. In the case of $\tau_{\mathrm{c} \pm} / \tau_{\mathrm{c} z}=5$, the bb sequence is more effective than the bp sequence for $\Delta t / \tau_{\mathrm{c}}=2^{-2}$, and is predominated by the bp sequence for $\Delta t / \tau_{\mathrm{c}}=2^{-3}$ and $2^{-4}$. In the case of $\tau_{\mathrm{c} \pm} / \tau_{\mathrm{c} z}=50$, 



Fig. 2. Time evolution of $\left|\tilde{\rho}_{10}\right|$ in the case of (a) $\tau_{\mathrm{c} \pm} / \tau_{\mathrm{c} z}=2$, (b) 5 and (c) 50 . The solid lines and dotted lines correspond to the cases of the bb sequence and the bp sequence, respectively, with $\Delta t / \tau_{\mathrm{c}}=2^{-2}, 2^{-3}$ and $2^{-4}$ from lower to upper lines. The dashed lines represent the case where $\pi$ pulses are absent.

the bb sequence is more effective than the bp sequence for $\Delta t / \tau_{\mathrm{c}}=2^{-2}$ and $2^{-3}$, and is as effective as the bp sequence in the shortest interval case of $\Delta t / \tau_{\mathrm{c}}=2^{-4}$. The above results indicate that the effectiveness of the pulse sequences depends on a relative strength of the two decoherence processes especially when a pulse interval is slightly shorter than $\tau_{\mathrm{c}}$. Only in the short-interval limit, the bp sequence is more effective than, or at least as effective as, the bb sequence regardless of $\tau_{\mathrm{c} \pm} / \tau_{\mathrm{c} z}$.

The above results are explained as follows. The bp sequence contains both phase-flips and bit-flips, and thus can cancel out the influence of both $\mathcal{H}_{z}$ and $\mathcal{H}_{ \pm}$, while the bb sequence can cancel out the influence of only $\mathcal{H}_{z}$. Thus, the bp sequence is much more effective than the bb sequence in the case of $\tau_{\mathrm{c} \pm} / \tau_{\mathrm{c} z} \sim 1$, where the influence of both $\mathcal{H}_{z}$ and $\mathcal{H}_{ \pm}$is important. However, the interval of two adjacent bit-flips in the bp sequence is equal to $2 \Delta t$, and is twice longer than that in the bb sequence. This indicates that the bp sequence reduces the influence of $\mathcal{H}_{z}$ less effectively than the bb sequence. Thus, in the case of $\tau_{\mathrm{c} \pm} / \tau_{\mathrm{c} z} \gg 1$, where $\mathcal{H}_{z}$ is more important than $\mathcal{H}_{ \pm}$, the bp sequence becomes less effective than the bb sequence unless $\Delta t$ is very much shorter than $\tau_{\mathrm{c} z}$.

In Fig. 3, we display the phase shift $\Delta \theta(t)$ for $\Delta t / \tau_{\mathrm{c}}=2^{-3}$ and $2^{-4}$ in the case of $\tau_{\mathrm{c} \pm} / \tau_{\mathrm{c} z}=2$. Obviously, $\Delta \theta(t) \equiv 0$ in the absence of qubit-environment interactions. We observe that the phase shift is suppressed by either of the bb or bp sequence. The phase shift in the cases of $\tau_{\mathrm{c} \pm} / \tau_{\mathrm{c} z}=5$ and 50 is very small, so we do not display the corresponding results.

\section{Summary and Conclusion}

To study the effectiveness of the pulse control, we have studied the time evolution of a qubit coupled with a quantum environment under a periodic $\pi$ pulses. We have considered a general interaction Hamiltonian which induces not only the pure dephasing but also the decoherence with population decay. We have examined the effectiveness of the bb sequence consisting of only bit-flips and the bp sequence consisting of bit- and phase-flips. We have derived the equation of motion for the reduced density matrix. The resulting equation is 

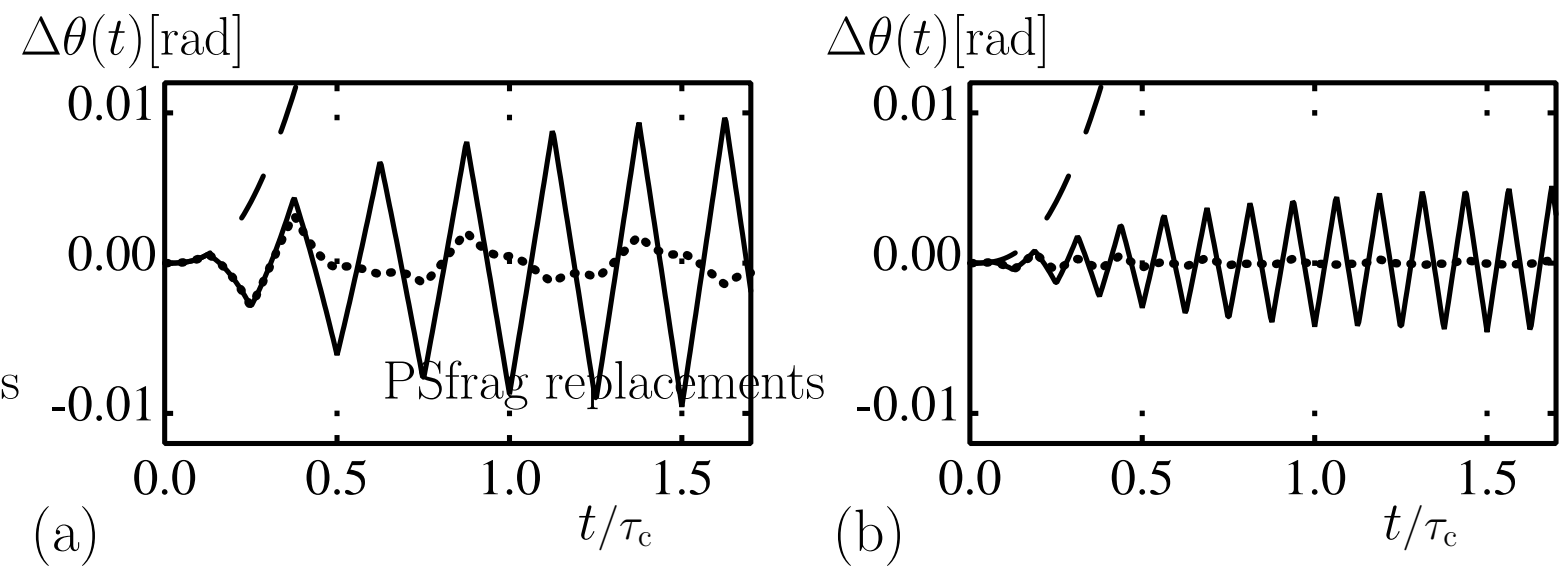

Fig. 3. Phase shift $\Delta \theta(t)$ in the case of $\tau_{\mathrm{c} \pm} / \tau_{\mathrm{c} z}=2$ with (a) $\Delta t / \tau_{\mathrm{c}}=2^{-3}$ and (b) $2^{-4}$. The solid lines and dotted lines correspond to the cases of the bb sequence and the bp sequence, respectively. The dashed lines represent the case where $\pi$ pulses are absent.

applicable to arbitrary sequences consisting of bit- and/or phase-flips.

By numerically solving the equation of motion, we have shown that the effectiveness of the pulse sequences depends on a relative strength of the two decoherence processes especially when a pulse interval is slightly shorter than $\tau_{\mathrm{c}}$. We have found that the bp sequence is more effective than, or at least as effective as, the bb sequence regardless of $\tau_{\mathrm{c} \pm} / \tau_{\mathrm{c} z}$ only in the short-interval limit. This means that if we cannot prepare periodic $\pi$ pulse with a short enough interval, the bp sequence is not necessarily the best choice unless $\tau_{\mathrm{c} \pm} / \tau_{\mathrm{c} z} \sim 1$.

\section{Acknowledgement}

The present authors thank Prof. M. Yamanishi for calling their attention to ref. 7. 


\section{References}

1) P. Tombesi and D. Vitali: Phys. Rev. A 51 (1995) 4913.

2) P. W. Shor: Phys. Rev. A 52 (1995) 2493.

3) A. M. Steane: Proc. R. Soc. Lond. A 452 (1996) 2551.

4) L. M. Duan and G. C. Guo: Phys. Rev. Lett. 79 (1997) 1953.

5) P. Zanardi and M. Rasetti: Phys. Rev. Lett. 79 (1997) 3306.

6) M. Ban: J. Mod. Opt. 45 (1998) 2315.

7) L. Viola and S. Lloyd: Phys. Rev. A 58 (1998) 2733.

8) G. S. Agarwal: Phys. Rev. A 61 (2000) 013809.

9) L. M. Duan and G. C. Guo: Phys. Lett. A 261 (1999) 139.

10) L. Viola, E. Knill and S. Lloyd: Phys. Rev. Lett. 82 (1999) 2417.

11) D. Vitali and P. Tombesi: Phys. Rev. A 59 (1999) 4178.

12) C. Uchiyama and M. Aihara: Phys. Rev. A 66 (2002) 32313.

13) V. Protopopescu, R. Perez, C. D. Helon and J. Schmulen: J. Phys. A 36 (2003) 2175.

14) T. Murakami and Y. Takane: J. Phys. Soc. Jpn. 74 (2005) Suppl. 265.

15) Y. Takane and T. Murakami: J. Phys. Soc. Jpn. 74 (2005) 2243.

16) U. Weiss: Quantum Dissipative Systems (World Scientific, Singapore, 1993) 2nd ed.

17) F. Shibata and T. Arimitsu: J. Phys. Soc. Jpn. 49 (1980) 891, and references therein. 